\title{
Anorexia nervosa and autism: a prospective twin cohort study
}

\author{
Lisa Dinkler, ${ }^{1}$ Mark J. Taylor, ${ }^{2}$ Maria Råstam, ${ }^{1,3}$ Nouchine Hadjikhani,,${ }^{1,4}$ \\ Cynthia M. Bulik, ${ }^{2,5,6}$ Paul Lichtenstein, ${ }^{2}$ Christopher Gillberg, ${ }^{1}$ and \\ Sebastian Lundström ${ }^{1}$
}

${ }^{1}$ Gillberg Neuropsychiatry Centre, Institute of Neuroscience and Physiology, University of Gothenburg, Gothenburg, Sweden; ${ }^{2}$ Department of Medical Epidemiology and Biostatistics, Karolinska Institutet, Stockholm, Sweden; ${ }^{3}$ Department of Clinical Sciences Lund, Lund University, Lund, Sweden; ${ }^{4}$ Athinoula A. Martinos Center for Biomedical Imaging, Massachusetts General Hospital, Harvard Medical School, Charlestown, MA, USA; ${ }^{5}$ Department of Psychiatry, University of North Carolina at Chapel Hill, Chapel Hill, NC, USA; ${ }^{6}$ Department of Nutrition, University of North Carolina at Chapel Hill, Chapel Hill, NC, USA

\begin{abstract}
Background: Anorexia nervosa (AN) and autism spectrum disorder (ASD) may be phenotypically and etiologically linked. However, due to the absence of prospective studies, it remains unclear whether the elevation of autistic traits in AN is evident in early childhood. Here, we prospectively investigated autistic traits before and after the first diagnosis of AN. Methods: In a population-based sample of 5,987 individuals (52.4\% female) from the Child and Adolescent Twin Study in Sweden, parents reported autistic traits at ages 9 and 18. AN and ASD diagnoses were retrieved from the Swedish National Patient Register. In addition, AN diagnoses were ascertained by parent-reported treatment for AN. We compared whether individuals with and without AN differed in autistic traits before the first diagnosis of AN (age 9) and after the first diagnosis of AN (age 18). Results: We did not find evidence for elevated autistic traits in 9-year-old children later diagnosed with AN. At age 18, however, there was a marked elevation in restricted/repetitive behavior and interests, but only in the subgroup of individuals with acute AN. A less pronounced elevation was observed for social communication problems. Conclusions: Coping strategies in individuals with ASD and the somewhat different female ASD phenotype may explain why we did not find elevated autistic traits in children who later developed AN. Alternatively, it is possible that elevated autistic traits were not present prior to the onset of AN, thus questioning the previously reported elevated prevalence of ASD in AN. Future studies should use tailored measurements in order to investigate whether autistic traits in individuals with AN are best conceptualized as an epiphenomenon of the acute AN phase or whether these symptoms indeed represent ASD as a clinically verifiable neurodevelopmental disorder. Keywords: Eating disorder; anorexia nervosa; autism spectrum disorders; longitudinal studies.
\end{abstract}

\section{Introduction}

Anorexia nervosa (AN) is a severe eating disorder, characterized by significantly low body weight, intense fear of gaining weight, and undue influence of weight and shape on self-evaluation (American Psychiatric Association, 2013). Autism spectrum disorder (ASD) is a pervasive neurodevelopmental disorder starting in infancy, defined by difficulties with social interaction and communication, and restricted/repetitive behavior and interests (RRBIs). Clinically, patients with AN are often described as socially withdrawn, displaying obsessive, rigid, and repetitive thoughts and behaviors - characteristics highly prominent in ASD as well (Zucker et al., 2007). AN and ASD have been reported to co-occur more than expected by chance (Westwood \& Tchanturia, 2017). A recent register-based cohort study reported an aggregation of ASD diagnoses in individuals with AN, where an individual with AN was 15 times more likely to be assigned a diagnosis of ASD than individuals without AN (Koch et al., 2015), and

Conflict of interest statement: See Acknowledgements for full disclosures. numerous studies have shown an overrepresentation of autistic traits in individuals with AN (for a review, see Westwood et al., 2016). Furthermore, AN and ASD co-aggregate within families, suggesting a common etiology of both disorders (Koch et al., 2015).

The autism phenotype is often much less evident in young girls, and many female ASD cases do not become apparent until they get in touch with health care for other (secondary) psychiatric disorders such as AN in adolescence or adult life (Gould \& AshtonSmith, 2011). Psychiatric comorbidities such as AN, anxiety, and depression can also obscure an underlying ASD and delay its diagnosis (Mandy \& Tchanturia, 2015; Trubanova, Donlon, Kreiser, Ollendick $\&$ White, 2013). While symptoms in early childhood are a prerequisite for an ASD diagnosis (American Psychiatric Association, 2013), AN has its typical onset in adolescence and requires no childhood symptomatology according to prevailing nosological systems (Micali, Hagberg, Petersen \& Treasure, 2013; Zerwas et al., 2015). However, there is a paucity of longitudinal studies examining ASD and autistic traits in childhood before the onset of AN. The nature of the co-occurrence of ASD in AN 
remains unclear as most previous studies examining their association are limited by their cross-sectional nature (for a review, see Westwood \& Tchanturia, 2017). Assessing autistic traits such as extreme rigidity or obsessive interests (e.g., in calories, body shape, or exercise) during the acute starved state could lead to an overestimation of the prevalence of ASD in AN.

A few studies have attempted to investigate childhood autistic traits in individuals with AN, but these have used retrospective parental reports, which are prone to bias (Gillberg \& Råstam, 1992, Pooni, Ninteman, Bryant-Waugh, Nicholls \& Mandy, 2012, Rhind et al., 2014, Westwood, Mandy, Simic \& Tchanturia, 2018; for a review, see Westwood \& Tchanturia, 2017). Finally, studies assessing ASD without taking information on childhood autistic traits into account report a much higher prevalence of individuals with AN meeting clinical cutoff for ASD (10\%-52.5\%) (Bentz et al., 2017; Postorino et al., 2017; Westwood, Mandy \& Tchanturia, 2017) than studies utilizing parental reports of autistic traits in childhood (4\%-10\%) (Gillberg \& Råstam, 1992; Pooni et al., 2012; Rhind et al., 2014; Westwood et al., 2018). Taken together, it is unclear whether the observed elevation of autistic traits in AN is present from childhood (i.e., trait) or whether it is primarily associated with the onset of AN (i.e., state).

In the present study, we, for the first time, prospectively investigated the association between ASD and AN, by comparing autistic traits in individuals with and without a later AN diagnosis at two time points: before AN onset (age 9) and after AN onset (age 18). Furthermore, we examined whether higher levels of autistic traits are specifically related to acute AN, by comparing the levels of autistic traits in those with and without acute AN at the time of assessment of autistic traits.

\section{Method}

Two sources of data were used in this study: (a) the Child and Adolescent Twin Study in Sweden (CATSS) and (b) the Swedish National Patient Register (NPR), providing both continuous and diagnostic measurements for ASD and diagnostic measurements for AN (Figure 1). The CATSS and its linkage to the NPR have ethical approval from the Regional Ethical Review Board in Stockholm (Dnr 02-289, 2010/597-31/1, 2016/ 2135-31 and 2010/322-31/2, 2010/1410-31/1). Written and/or oral consent was given by the parents at twin ages 9 and 18 .

\section{Participants - The Child and Adolescent Twin Study in Sweden}

CATSS is an ongoing longitudinal study aiming to include all twins born in Sweden since July 1, 1992 (Anckarsäter et al., 2011). The baseline assessment is conducted in connection with the twins' $9^{\text {th }}$ birthday (the cohorts of 1992-1995 were assessed at age 12, CATSS-9), including parent-reports only. When the twins turn 18 , a follow-up study is conducted via a Web questionnaire (CATSS-18). In CATSS-18, parents and twins are assessed regarding the somatic and mental health of the twins. The answering frequency of parents in CATSS-9 and CATSS- 18 is $75.8 \%$ and $41.4 \%$, respectively. A total of $47.4 \%$ of the parents in CATSS-9 also responded in CATSS-18. An attrition analysis can be found in the results section. The present study includes twins born 1992-1999, whose parents had responded to the Autism-Tics, AD/HD, and other Comorbidities inventory (A-TAC) at both 9 and $18(n=7,272)$. We excluded individuals with congenital or early brain damage syndromes and known chromosomal syndromes $(n=73)$, and individuals with any missing items on the A-TAC at age 9 or 18 $(n=1,212)$. The final sample consisted of 5,987 individuals (52.4\% female).

\section{Measurements}

Autistic traits. CATSS-9 contains the A-TAC, which is a fully structured parental telephone interview designed for large-scale epidemiological research (Hansson et al., 2005). It covers all common child and adolescent neurodevelopmental and psychiatric disorders. A-TAC items are scored with 'no' (0),

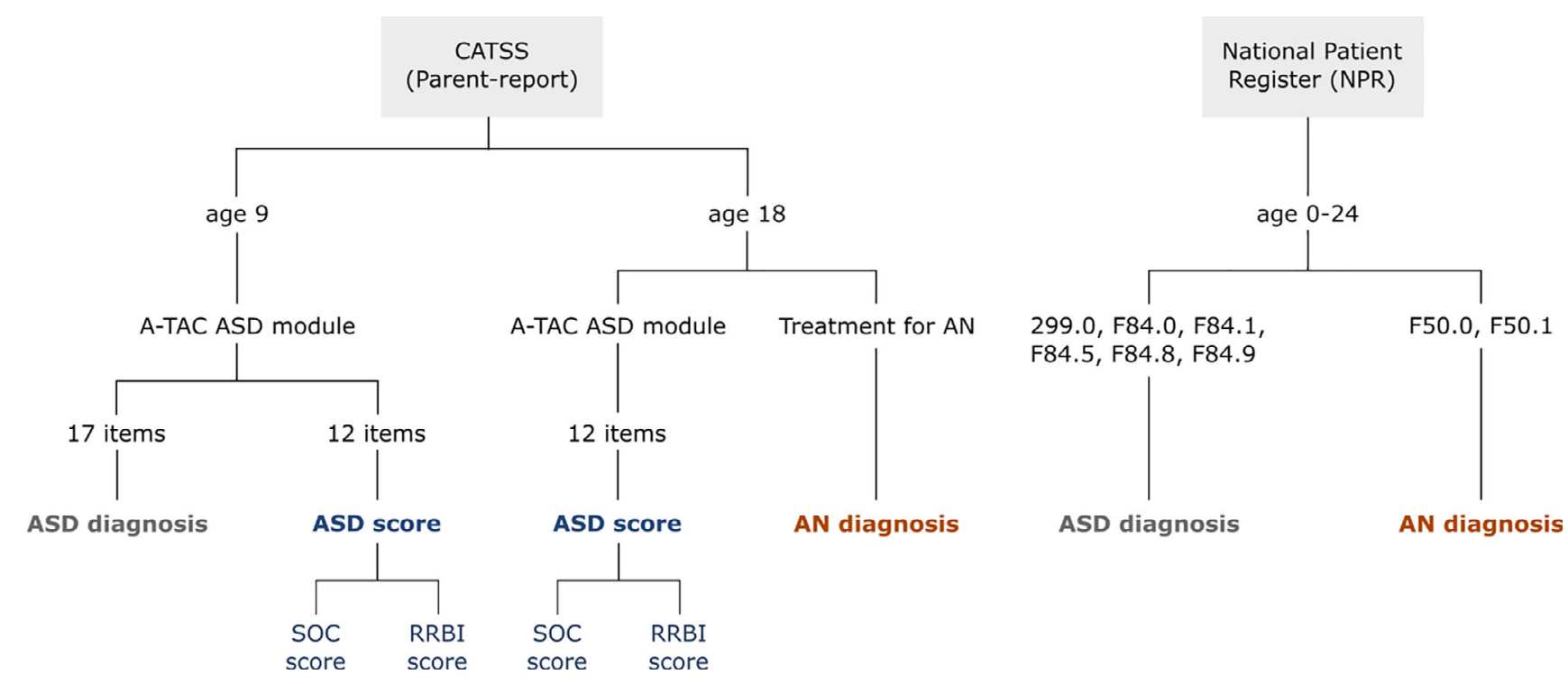

Figure 1 Overview of measurements employed in the study. AN, Anorexia nervosa; ASD, Autism spectrum disorder; A-TAC, Autism-Tics, AD/HD, and other Comorbidities inventory; CATSS, Child and Adolescent Twin Study in Sweden; RRBI, Restricted/repetitive behavior and interests; SOC, Social communication. F50.0, F50.1, 299.0, F84.0, F84.1, F84.5, F84.8, F84.9: ICD-9/ICD-10 diagnostic codes 
'yes, to some extent' (0.5), or 'yes' (1) and are added up to a sum score in each module. The A-TAC has been validated against clinical diagnoses in cross-sectional (Hansson et al., 2005; Larson et al., 2010) and longitudinal studies (Larson et al., 2013; Mårland et al., 2017). The ASD module in CATSS-9 consists of 17 items modeled around the DSM-IV criteria and well-known clinical characteristics. It yields an ASD score with a minimum of 0 and a maximum of 17 . We used the validated cutoff score of $\geq 8.5$ to identify individuals with a screening diagnosis of ASD at age 9 (sensitivity .71/specificity .95) (Larson et al., 2010).

In order to measure autistic traits continuously, we used the ASD score. In CATSS-18, only 12 of the 17 ASD items were included (the 12 items pertain to the DSM-IV criteria). To make the ASD scores comparable across ages 9 and 18, we used the same 12 items included at age 18 to calculate the ASD score at age 9 (instead of all 17 items). The ASD score was split into two subscales that align with the DSM- 5 criteria: social communication (8 items) and RRBIs (4 items; Figure 1). In both CATSS-9 and CATSS-18, parent-reports were used in order to minimize rater effects. The ASD score at age 18 has not yet been validated against ASD diagnoses from the NPR. Therefore, we calculated the area under the curve for ASD score and ASD diagnosis ( 81 cases). The area under the curve at age 18 was .90 (age $9=.80$ ), suggesting the ASD score at age 18 is a valid measurement for ASD diagnosis. A list of the ASD items in the $\mathrm{A}-\mathrm{TAC}$ can be found in Table S1 or at https://gnc.gu.se/ english/research/screening-questionnaires/a-tac-screeningquestionnaire.

Registered diagnoses of AN and ASD. The CATSS is linked to the Swedish NPR, which contains diagnoses from psychiatric inpatient care from 1973 onward and data from specialized outpatient care from 2001 onward. The linkage with CATSS includes NPR data up until December 31, 2016; therefore, the follow-up age for NPR diagnoses in our sample differed by year of birth (age range: 17-24 years). The following diagnostic codes (including date of diagnosis) were retrieved for all 5,987 participants: AN: ICD-10: F50.0, F50.1; ASD: ICD-9: 299.0, ICD-10: F84.0, F84.1, F84.5, F84.8, F84.9. Importantly, the diagnosis F50.1 (atypical AN) in the ICD-10 is typically given when the amenorrhea criterion is not present. This differs from the DSM-5 diagnosis of atypical AN that is given if the individual meets all criteria for AN except low weight. Individuals with DSM- 5 atypical AN are not captured in this analysis. AN in the NPR has not been subjected to formal reliability/validity testing; however, high validity of the NPR has been reported for a range of neurodevelopmental and psychiatric disorders, including ASD (Idring et al., 2012; Ludvigsson et al., 2011; Rück et al., 2015). For each individual with an NPR diagnosis of AN, we defined the age of onset as the age at first diagnosis.

Classification of individuals with lifetime AN. A total of 41 girls $(1.3 \%)$ and 5 boys $(0.2 \%)$ had diagnoses of AN in the NPR. Of these 46 individuals, 44 received their first AN diagnosis before the CATSS- 18 assessment, and 2 within six months after CATSS-18. In addition to the NPR diagnoses (i.e., even if no NPR diagnosis was present), we included parentreports of AN treatment to identify individuals with AN. In CATSS-18, parents were asked: 'Has the twin been treated for AN?'. If the parent responded 'Yes, earlier' or 'Yes, now', the twin was identified as having/having had AN (Figure 1). Parent-reported treatment data were available for $99.5 \%$ of the sample. AN was identified in additional 35 girls and 15 additional boys by parent-report. In total, 76 girls and 20 boys were identified with lifetime AN (Figure 2).

Classification of individuals with acute AN versus history of $A N$. In a second step, we divided girls with lifetime AN (we were underpowered to do this for boys) into two groups based on their AN status at the time of CATSS-18 (i.e., during the assessment of autistic traits at age 18), using the following stepwise procedure: Girls were identified with acute $A N$ (a) if their parents reported current treatment (as opposed to earlier treatment) for AN in CATSS-18 (answer option 'Yes, now', $n=15$ ), (b) if they had received an AN diagnosis in the NPR within 6 months before or after the CATSS-18 questionnaire, but parents did not report current treatment $(n=3)$, and (c) if (a) and (b) did not apply, but they had a self-reported BMI lower than $17^{1}$ during CATSS-18 (besides being identified with lifetime AN; $n=6$ ). Following this algorithm, girls were excluded from this classification if parents reported previous (but not current) treatment and no NPR data were available $(n=30)$, since we could not reliably ascertain their AN status during CATSS-18. In total, we identified 24 girls with acute AN and 22 girls with a history of AN at the time of CATSS-18 (Figure 2).

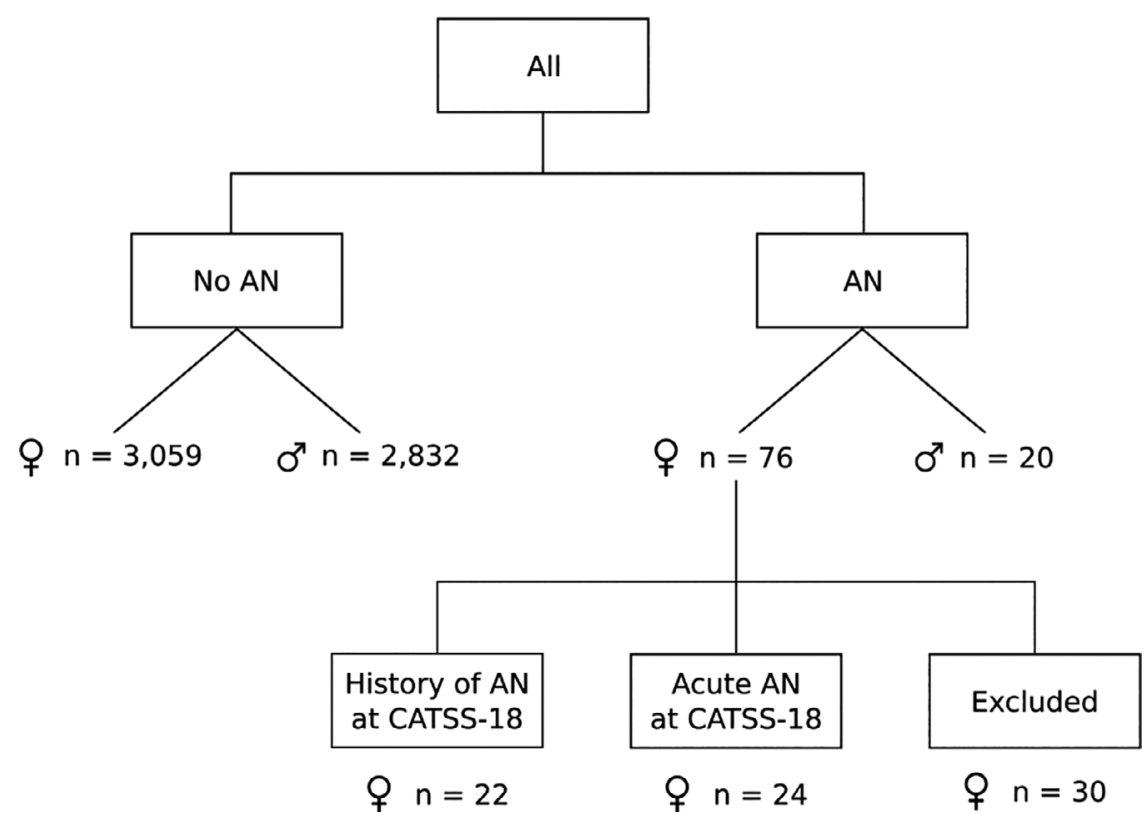

Figure 2 Groups within the sample. AN, Anorexia nervosa; CATSS-18, Child and Adolescent Twin Study in Sweden, assessment at age 18

(C) 2020 The Authors. Journal of Child Psychology and Psychiatry published by John Wiley \& Sons Ltd on behalf of Association for Child and Adolescent Mental Health. 


\section{Statistical analyses}

In a first step, we compared the ASD scores at ages 9 and 18 between individuals with lifetime $\mathrm{AN}$ and individuals without AN. This was done separately for boys and girls. In a second step, we excluded all boys due to the low number of boys with AN $(n=20)$, and then compared the ASD score at age 18 between girls with a history of AN and girls with acute AN. We used generalized estimating equations (GEE) in order to account for the correlation between twins in a pair (i.e., nonindependent observations) (Carlin, Gurrin, Sterne, Morley $\&$ Dwyer, 2005). The robust sandwich estimator in GEE treats each twin pair as a cluster and produces valid standard errors even if the within-group correlations are mis-specified (StataCorp, 2017a). The ASD score was extremely skewed (boys/ girls: age 9 skew $=5.0 / 6.6$, age 18 skew $=3.2 / 3.4)$ and logtransformation failed; therefore, a negative binomial distribution with log link function was used in the GEE models. When using log links, parameter estimates are expressed and discussed as incidence rate ratios (IRR). In the case of a binary predictor, the IRR can be interpreted similar to an odds ratio. Data management was performed in SAS 9.4, and data analysis was performed in R 3.5.3 (R Core Team, 2019) and Stata 15.0 (StataCorp, 2017b).

\section{Results}

\section{Descriptive statistics}

The lifetime prevalence of AN was $2.4 \%$ in girls $(n=76)$ and $0.7 \%$ in boys $(n=20)$. The average age of AN onset (measured as age at first diagnosis in the NPR) was similar in girls and boys (Table S2). A total of 31 girls (1\%) and 50 boys (1.8\%) had diagnoses of ASD in the NPR. The median age of first ASD diagnosis was almost 3 years higher in girls than in boys (Table S2). Only two of the 31 girls with ASD $(6.5 \%)$ were diagnosed before the age of 10 , compared to $14 \%$ of boys.

The overlap of AN and ASD diagnoses was low ( $n=$ 1 ), corresponding to an ASD prevalence of $1.3 \%$ in girls with $\mathrm{AN}$ and $0 \%$ in boys with AN. Using the validated diagnostic proxy for the A-TAC at age 9, one boy had ASD and a diagnosis of AN in the NPR. This corresponds to an ASD prevalence of $5 \%$ in boys with $\mathrm{AN}$ and $0 \%$ in girls with $\mathrm{AN}$.

\section{ASD score at ages 9 and 18 in girls}

Girls with AN did not show elevated total ASD scores at age 9 compared to the population of girls who did not develop AN $(M=0.23$ in both groups, IRR $=1.04$, 95\% CI 0.59-1.82, $p=.892$, Table 1, Figure 3). No significant differences emerged in the ASD subscales at age 9 . In contrast, at age 18, girls with AN had a 1.48 times higher score in social communication symptoms and a 2.36 times higher score in RRBI symptoms compared to girls without AN.

Table 1 Score on the autism module of the Autism-Tics, AD/HD, and other Comorbidities inventory (A-TAC) at age 9 and age 18 by sex and anorexia nervosa (AN) status

\begin{tabular}{|c|c|c|c|c|c|c|c|c|c|}
\hline & \multicolumn{3}{|c|}{ Age 9} & \multicolumn{6}{|c|}{ Age 18} \\
\hline & $\begin{array}{l}\text { No AN } \\
M(S D)\end{array}$ & $\begin{array}{c}\text { AN } \\
M(S D)\end{array}$ & $\begin{array}{l}\text { AN versus } \\
\text { No AN } \\
\text { IRR ( } 95 \% \\
\text { CI) }\end{array}$ & $\begin{array}{l}\text { No AN } \\
M(S D)\end{array}$ & $\begin{array}{c}\text { AN } \\
M(S D)\end{array}$ & $\begin{array}{l}\text { AN versus } \\
\text { No AN } \\
\text { IRR (95\% } \\
\text { CI) }\end{array}$ & $\begin{array}{l}\text { History } \\
\text { of AN } \\
M(S D)\end{array}$ & $\begin{array}{l}\text { Acute } \\
\text { AN } \\
M(S D)\end{array}$ & $\begin{array}{c}\begin{array}{c}\text { Acute AN versus } \\
\text { History } \\
\text { of } \mathrm{AN}^{\mathrm{a}}\end{array} \\
\text { IRR }(95 \% \mathrm{CI})\end{array}$ \\
\hline Girls & $n=3,059$ & $n=76$ & & $n=3,059$ & $n=76$ & & $n=22$ & $n=24$ & \\
\hline Total ASD Score & $\begin{array}{l}0.23 \\
(0.70)\end{array}$ & $\begin{array}{l}0.23 \\
(0.59)\end{array}$ & $\begin{array}{l}1.04(0.59 \\
1.82)\end{array}$ & $\begin{array}{l}0.61 \\
(0.98)\end{array}$ & $\begin{array}{l}1.04 \\
(1.40)\end{array}$ & $\begin{array}{l}1.72(1.23 \\
2.38)\end{array}$ & $\begin{array}{l}0.52 \\
(1.01)\end{array}$ & $\begin{array}{l}1.56 \\
(1.25)\end{array}$ & $\begin{array}{l}2.99(1.27 \\
7.03)\end{array}$ \\
\hline $\begin{array}{l}\text { Social } \\
\text { communication }\end{array}$ & $\begin{array}{l}0.14 \\
(0.47)\end{array}$ & $\begin{array}{l}0.11 \\
(0.33)\end{array}$ & $\begin{array}{l}0.85(0.43 \\
1.67)\end{array}$ & $\begin{array}{l}0.45 \\
(0.75)\end{array}$ & $\begin{array}{l}0.64 \\
(0.96)\end{array}$ & $\begin{array}{l}1.48(1.04 \\
2.10)\end{array}$ & $\begin{array}{l}0.39 \\
(0.87)\end{array}$ & $\begin{array}{l}0.81 \\
(0.76)\end{array}$ & $2.10(0.77,5.73)$ \\
\hline $\begin{array}{l}\text { Restricted/ } \\
\text { repetitive } \\
\text { behavior } \\
\text { and interests }\end{array}$ & $\begin{array}{l}0.09 \\
(0.34)\end{array}$ & $\begin{array}{l}0.12 \\
(0.38)\end{array}$ & $\begin{array}{l}1.30(0.65 \\
2.60)\end{array}$ & $\begin{array}{l}0.16 \\
(0.41)\end{array}$ & $\begin{array}{l}0.40 \\
(0.61)\end{array}$ & $\begin{array}{l}2.36(1.60, \\
3.48)\end{array}$ & $\begin{array}{l}0.14 \\
(0.23)\end{array}$ & $\begin{array}{l}0.75 \\
(0.74)\end{array}$ & $\begin{array}{l}5.50(2.49 \\
12.14)\end{array}$ \\
\hline Boys & $n=2,832$ & $n=20$ & & $n=2,832$ & $n=20$ & & & & \\
\hline Total ASD Score & $\begin{array}{l}0.40 \\
(0.95)\end{array}$ & $\begin{array}{l}0.63 \\
(1.42)\end{array}$ & $\begin{array}{l}1.69(0.68 \\
4.18)\end{array}$ & $\begin{array}{l}0.74 \\
(1.08)\end{array}$ & $\begin{array}{l}1.18 \\
(1.90)\end{array}$ & $\begin{array}{l}1.91(0.97 \\
3.74)\end{array}$ & & & \\
\hline $\begin{array}{l}\text { Social } \\
\text { communication }\end{array}$ & $\begin{array}{l}0.22 \\
(0.62)\end{array}$ & $\begin{array}{l}0.38 \\
(0.89)\end{array}$ & $\begin{array}{l}1.84(0.76 \\
4.44)\end{array}$ & $\begin{array}{l}0.54 \\
(0.81)\end{array}$ & $\begin{array}{l}0.68 \\
(1.05)\end{array}$ & $\begin{array}{l}1.60(0.86 \\
2.98)\end{array}$ & & & \\
\hline $\begin{array}{l}\text { Restricted/ } \\
\text { repetitive } \\
\text { behavior } \\
\text { and interests }\end{array}$ & $\begin{array}{l}0.18 \\
(0.45)\end{array}$ & $\begin{array}{l}0.25 \\
(0.60)\end{array}$ & $\begin{array}{l}1.49(0.53 \\
4.20)\end{array}$ & $\begin{array}{l}0.20 \\
(0.44)\end{array}$ & $\begin{array}{l}0.50 \\
(0.96)\end{array}$ & $\begin{array}{l}2.65(1.13 \\
6.20)\end{array}$ & & & \\
\hline
\end{tabular}

Statistical method: Generalized estimating equations (GEE) with negative binomial distribution, log link function, and exchangeable within-cluster correlation structure; standard errors adjusted for 2,168 (girls)/2,031 (boys) clusters (i.e., twin pairs). IRR, Incidence Rate Ratio; CI, Confidence Interval.

${ }^{a}$ Since there were no complete twin pairs (i.e., clusters) included in this analysis, standard errors were not adjusted for clusters (i.e., an independent within-cluster correlation structure was used). 


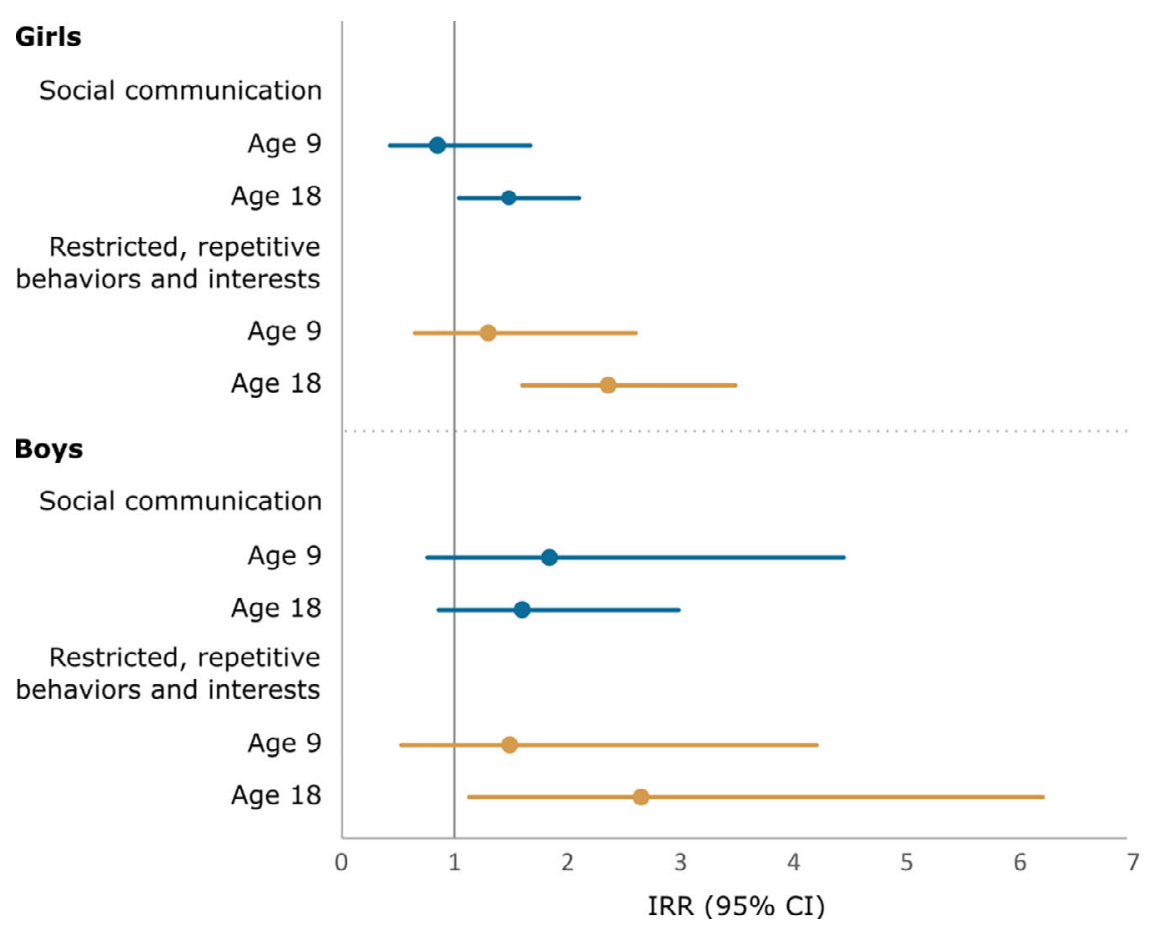

Figure 3 Incidence Rate Ratios (IRRs) comparing the ASD subscale scores in AN versus no AN at age 9 and age 18. Compare Table 1

\section{Item-based analysis at ages 9 and 18 in girls}

In an exploratory analysis, we assessed each ASD item separately, in order to investigate which autistic traits were specifically characteristic of girls with AN. At age 9, the items with the highest IRRs (albeit nonsignificant) in girls who would later develop AN, compared to girls who would not, were on the social communication subscale: 'Does he/she like to repeat words and expressions or does he/she use words in a way other people find strange?' (IRR $=2.24,95 \%$ CI 0.74-6.74) and 'Does he/she have difficulties with games of make-believe or does he/she imitate others considerably less than other children?' (IRR $=2.45$, 95\% CI 0.65-9.23). The IRRs of the other ten ASD items were lower than 2 , or even than lower than 1 .

At age 18, the items with the highest significant elevation in girls with lifetime AN, compared to girls without lifetime AN, were 'Does he/she get absorbed by routines in such a way as to produce problems for him/herself or others?' (IRR $=6.87,95 \%$ CI 4.1311.43), 'Does he/she like to repeat words and expressions or does he/she use words in a way that other people find strange?' (IRR $=2.97,95 \%$ CI 1.07 8.19), and 'Does he/she get absorbed by his/her interests in such a way as being repetitive or too intense?' (IRR $=2.71,95 \%$ CI 1.48-4.96).

\section{ASD score at ages 9 and 18 in boys}

At age 9, we found no significant differences between boys with and without AN (Table 1, Figure 3). Similar to girls, boys with AN had significantly more RRBI symptoms at age 18 compared to males without AN; however, the power for boys was low ( $n$ $=20$ boys with $\mathrm{AN}$ ).

\section{$A S D$ score at age 18 in girls with acute $A N$ versus history of $A N$}

Girls with acute AN had higher total ASD scores at age 18 compared to girls with a history of AN (IRR $=2.99$, Table 1$)$. They had higher scores on both subscales, albeit the difference for social communication symptoms was nonsignificant. These results are graphically presented in individual-based spaghetti plots that can be found in Figure 4. No significant differences were observed between the group with history of AN and the population of girls without AN on either of the scales (social communication: 0.39 vs. 0.45 ; IRR $=0.86,95 \%$ CI $0.34-2.17$; RRBI: 0.14 vs. 0.16 ; IRR $=0.84,95 \%$ CI $0.42-1.67$, Table 1).

\section{Post hoc power analysis}

Since the numbers of individuals with AN were limited, we conducted a post hoc power analysis using G*Power 3.1.9.4 (Faul, Erdfelder, Lang \& Buchner, 2007) in order to compute the minimum detectable effect sizes (mean difference in total ASD score between AN/no AN groups) given an $\alpha$ of .05, a power of .80 , and the sample sizes ${ }^{2}$. In girls, the minimum detectable Cohen's $d$ was 0.29 (corresponding points on the total ASD score: $0.2^{3}$ at age 9 and 0.3 at age 18). In boys, our tests were powered to detect a Cohen's $d$ of 0.56 (corresponding points on the total ASD score: 0.5 at age 9 and 0.6 at age 18). 

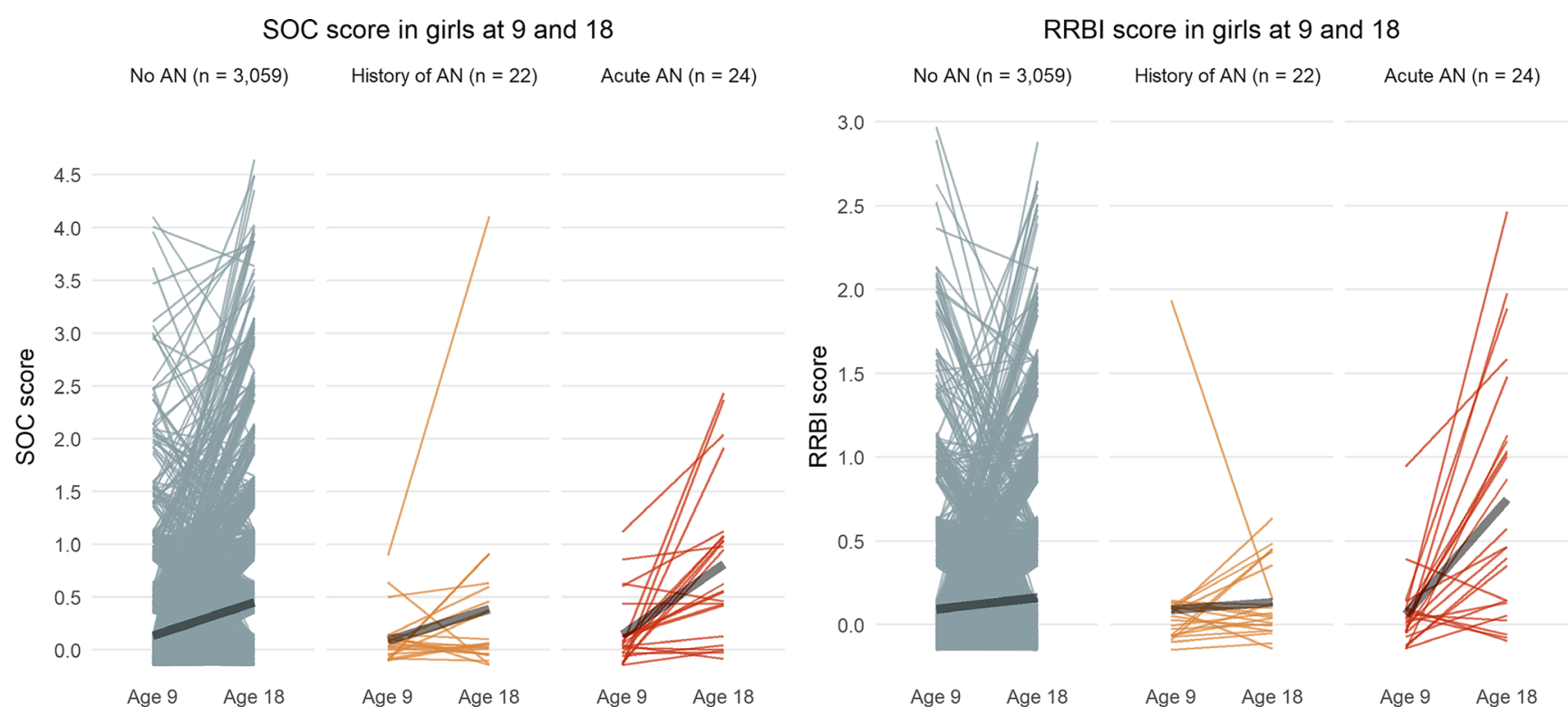

Figure 4 Within-individual change in ASD subscale scores from age 9 to age 18 by group. Each colored line corresponds to one individual. The thicker black line is the group average. Compare Table 1. AN, Anorexia nervosa; SOC, Social communication; RRBI, Restricted/ repetitive behavior and interests

In the subanalysis of girls with lifetime $\mathrm{AN}$, we were powered to detect a Cohen's $d$ of 0.75 (corresponding points on the total ASD score: 0.9).

\section{Attrition analysis}

The attrition rate from CATSS- 9 to CATSS-18 was $52.6 \%$. In an attrition analysis, we found that compared to the children of responding parents at age 18 - the children of nonresponding parents $(n=$ 8,054 , of those 3,760 female) had a similar prevalence of registered AN diagnoses (girls: 1.73\%, boys: $0.14 \%$ ), but more registered ASD diagnoses (girls: 1.54\%, boys: $3.52 \%$ ) and higher ASD scores at age 9 in general. However, similar to the primary analyses the nonresponders at age 18 with and without a later AN diagnosis did not differ in their ASD scores at age 9 (girls: $M=0.31$ vs. $M=0.32$; IRR $=0.97,95 \% \mathrm{CI}$ $0.46-2.05, n_{\mathrm{AN}}=65$; boys: $M=0.5$ vs. $M=0.62$; IRR $=0.81,95 \%$ CI $\left.0.18-3.75, n_{\mathrm{AN}}=6\right)$, suggesting that attrition did not bias our results.

\section{Discussion}

Using a prospective design of nearly 6000 individuals, we explored whether individuals with and without AN differed in autistic traits in childhood (before the onset of AN) and in late adolescence (after the onset of AN). Furthermore, we examined whether autistic traits at age 18 differed in girls with acute AN compared to those with a history of AN. We found no evidence for elevated autistic traits in children who later were diagnosed with AN. At age 18, however, markedly higher levels of RRBI symptoms were observed in individuals who had been diagnosed with AN at any point in their life. In girls (where we had power to do subanalyses), this increase was confined to the girls with acute AN. The same pattern, but less pronounced, could be seen for social communication symptoms.

Autistic traits in childhood were not overrepresented in children who were later diagnosed with AN, and only one of the 96 individuals with AN in this sample had a registered ASD diagnosis. This finding does not seem to be in line with the reported overrepresentation of ASD in AN by previous studies that examined registered diagnoses (Koch et al., 2015) or included retrospective parental reports on childhood autistic traits and therefore ascertained that autistic traits were present before the onset of AN (Gillberg \& Råstam, 1992; Rhind et al., 2014; Westwood et al., 2018). These reports would have suggested that we would find elevated autistic traits and a higher prevalence of ASD in individuals who would later develop AN; however, that was not the case in this sample. We did observe, however, elevated ASD scores, especially RRBIs, in individuals experiencing acute $\mathrm{AN}$, which is in line with previous studies (for a review, see Westwood et al., 2016) Several potential explanations exist for these results.

First, one may speculate that autistic traits are indeed present premorbidly in a subgroup of individuals with $\mathrm{AN}$; however, they become exacerbated and thereby detectable after the emergence of AN. In recent years, the concept of 'camouflaging' in cognitively able (i.e., without intellectual disability) individuals with ASD has been studied extensively. The AN + ASD subgroup is likely to be characterized by high achievement and perfectionism, as these are common in individuals with AN (Claydon \& Zullig, 2019; Dahlenburg, Gleaves \& Hutchinson, 2019; Sundquist, Ohlsson, Winkleby, Sundquist \& Crump, 
2016). Camouflaging comprises coping strategies to either hide behaviors associated with ASD or to purposely perform behaviors deemed to be neurotypical, in order to socially fit in. Examples of camouflaging are forcing eye contact, overemphasizing facial expressions or gestures, pre-preparing phrases and jokes, and memorizing social scripts (Hull et al., 2017; Lai et al., 2016). These strategies are used by children as young as 5 to 11 years (Dean, Harwood \& Kasari, 2017; Rynkiewicz et al., 2016). Camouflaging is very demanding and often takes its toll in form of exhaustion, depression, and anxiety (Hull et al., 2017; Lai et al., 2016). In individuals with unrecognized ASD who camouflage, the emergence of AN might finally lead to a depletion of resources to handle social demands, which in turn makes the autistic traits more noticeable. This is in line with the DSM-5 ASD criterion C that 'symptoms must be present in the early developmental period (but may not become fully manifest until social demands exceed limited capacities, or may be masked by learned strategies in later life)' (American Psychiatric Association, 2013).

A related issue is the assessment of ASD in females in general. Camouflaging is more common in females with ASD than in males with ASD (Hull et al., 2019; Lai et al., 2016). This has been proposed as one of the reasons why girls are less frequently diagnosed with ASD than boys, despite similar functioning (Kirkovski, Enticott \& Fitzgerald, 2013). It might also have contributed to the fact that the DSM diagnostic criteria as well as ASD questionnaires are almost entirely based on observations of males with ASD, whereas it has been repeatedly reported that females with ASD commonly present with different symptom profiles than males with ASD. For instance, girls with ASD not only show fewer RRBIs than boys with ASD (Van Wijngaarden-Cremers et al., 2014), but also qualitatively different RRBIs (Hiller, Young \& Weber, 2014). Furthermore, girls with ASD more commonly than boys engage in imaginative play, form extreme clingy attachments, interact mainly with younger children, and imitate the speech and movements of others (however, without a deeper understanding of the social situation) (Dean et al., 2017; Hiller et al., 2014; Kopp \& Gillberg, 2011). It has recently been argued that males and females should not be subjected to the same thresholds in the ASD screening instrument used in this study (A-TAC) and that different symptoms should be weighted according to sex, but no concrete suggestions to do so exist to date (Lundström et al., 2019). Thus, it is possible that more fine-grained examinations; for instance, questionnaires designed to take the 'female phenotype' of ASD into account, like the Autism Spectrum Screening Questionnaire-Revised Extended Version (ASSQREV) (Kopp \& Gillberg, 2011), might yield different results at age 9. Our findings from the item-level analysis suggest that childhood symptoms highly characteristic for typical Asperger syndrome (i.e., atypical use/repetition of words and expressions; difficulties with pretend play/imitation) might be predictive of later onset of AN in a subgroup of girls with ASD and AN. It has furthermore been suggested that parent-observed autistic traits in girls only become more overt from 10 years onward. While subtle social difficulties in childhood might have gone unnoticed by the parents, these difficulties escalate in adolescence, where the social contexts become more complex (Mandy, Pellicano, St Pourcain, Skuse \& Heron, 2018). In summary, camouflaging in cognitively able individuals with $\mathrm{ASD}$, the somewhat different female ASD phenotype and a possible later 'onset' of autistic traits in females may be reasons why we did not find elevated autistic traits in 9-yearolds who later developed AN compared to 9-year-olds who did not develop AN.

An alternative explanation is that the association between AN and ASD is not actually as strong as previously reported/suspected, or is confined to a particular subgroup (Westwood \& Tchanturia, 2017). In our study, elevated autistic traits (especially RRBIs) at age 18 were only evident in individuals with acute AN, while the level of autistic traits in the group with a history of AN was on par with the level in the general population. At first glance, this result seems to indicate that the association between AN and ASD might previously have been overestimated. ASD is a neurodevelopmental disorder requiring symptoms to be present from early childhood (American Psychiatric Association, 2013). Our result that autistic traits at age 18 were only elevated in individuals with acute AN suggests that the ASD score might actually have been detecting traits associated with the acute phase of AN. Indeed, the four RRBI items ${ }^{4}$ might have been rated by the parents with specific obsessions regarding food, weight/shape, or exercise in mind, which are typical in AN. On the other hand, as discussed above, a subgroup of individuals might exist in whom autistic traits have indeed been present since early life, but not noticeable due to successful camouflaging, with resources being exhausted with the onset of $\mathrm{AN}$, leading to the exacerbation of autistic traits. Parental accounts in clinical practice often do not identify premorbid concerns beyond a tendency to perfectionism and eagerness to please. Interpersonal or social difficulties may be cited as a trigger for AN, but not as a feature of early childhood. Two of the members of our research group (Gillberg, Råstam) have extensive (35-45 years) clinical follow-up experience of individuals with $\mathrm{AN}$ and/or $\mathrm{ASD}$, and of interacting with and interviewing their families. We observed that many parents of children/adolescents with AN were surprised when their child, who they felt never showed any problems, suddenly came down with the devastating symptoms of AN. In our experience, the eating problems were there long before the AN signs became severe. We also believe that, in many cases, the early childhood autistic 
traits were interpreted by parents as 'she does not have the time for such things, she is such a good, perfect, girl, stubborn yes, but...'

Clinically, it is of high value to identify underlying ASD in individuals with AN. ASD in AN is associated with higher eating disorder pathology and comorbidity at admission (Stewart, McEwen, Konstantellou, Eisler \& Simic, 2017; Tchanturia, Adamson, Leppanen \& Westwood, 2019) and poorer treatment outcome of traditional AN treatments (Stewart et al., 2017; Tchanturia, Larsson \& Adamson, 2016). Cognitive characteristics strongly associated with ASD, such as rigidity, inflexibility, and social processing difficulties, have been suggested to be maintaining factors for AN (Treasure, 2013; Treasure \& Schmidt, 2013), which is an understanding shared by patients with AN and ASD (Kinnaird, Norton, Stewart \& Tchanturia, 2019). Treatment methods addressing these maintenance factors have been developed for AN (Tchanturia, Doris, Mountford \& Fleming, 2015; Tchanturia, Lounes \& Holttum, 2014), but have yet to be evaluated more extensively for AN + ASD (Adamson, Leppanen, Murin \& Tchanturia, 2018; Tchanturia et al., 2016). In a recent qualitative study, Kinnaird et al. (2019) identified further potential adaptations to AN treatment that might improve treatment outcome in individuals with ASD, such as recognizing and diagnosing the underlying ASD, exploring the role of autistic traits in the eating pathology together with the clinician, taking sensory difficulties into account (e.g., during refeeding and noisy group treatment settings), and using alternative methods of communication (non-face-to-face).

However, as has been previously discussed (Mandy $\&$ Tchanturia, 2015), our results suggest that the assessment of ASD during the acute phase of AN requires extremely careful consideration: On the one hand, the acute AN phase may be when the ASD symptomatology becomes obvious, but on the other hand it is unclear whether this symptomatology indeed reflects autistic traits, rather than RRBIs associated with food, weight/shape, and exercise. This further highlights the importance of collecting detailed information on autistic traits in early development, when AN is present. In a population-based study capitalizing on detailed clinical examinations, a prevalence of $12 \%$ of ASD in AN has been reported (Anckarsäter et al., 2012). This prevalence was ascertained through in-depth examinations repeated over a period of 18 years, indicating that ASD in AN is not easily captured by questionnaires. Clinicians assessing ASD should be particularly attentive to possible AN, if RRBIs around food, weight/shape, and exercise constitute the bulk of the restricted/repetitive patterns of behavior, interests, or activities.

\section{Strengths and limitations}

This study has several strengths. It is a large-scale longitudinal and population-based sample, autistic traits were measured with a validated instrument, and the AN diagnoses were retrieved from a national register containing clinical diagnoses. Additionally, we used parent-reports of treatment for AN to adjust for a possible under-reporting of AN diagnoses in the NPR. This is one of the few studies including boys, which are underrepresented in the AN-ASD literature.

Nevertheless, the results should be considered in the light of some limitations. First, although our sample was large, the number of individuals with AN was low (especially for males) due to the low prevalence of this disorder ( 1\%). Second, our sample was 17-24 years old when AN diagnoses were extracted from the NPR and 18 years old when parents reported on treatment for AN. Although the majority of individuals with AN are diagnosed in adolescence (Zerwas et al., 2015), some individuals in the younger birth cohorts may not yet have developed $\mathrm{AN} /$ received an AN diagnosis at the time the data were extracted from the NPR. This could have led to false-negative classification and type II error. Future studies should include longer follow-up times for AN diagnoses. Mis-classification might also have occurred when dividing into the subgroups of acute AN and history of AN. Third, it has been suggested that ASD might genetically be more closely associated with the restricting subtype of AN than with the binge-eating/purging subtype (Watson et al., 2019). As AN subtypes are not documented in the NPR, we were unable to distinguish between them, precluding investigation of possible differences in their association with ASD. Finally, our sample consisted of twins; however, the generalizability to the general population is likely not affected for several reasons. It is not well known whether and to which degree AN is overrepresented in twins. One study has suggested a slightly higher risk of AN for twins compared to singletons (Goodman, Heshmati, Malki \& Koupil, 2014). The prevalence of AN in our sample was $2.4 \%$ for girls and $0.7 \%$ for boys, which is on par with previously reported population prevalence (KeskiRahkonen \& Mustelin, 2016; Micali et al., 2017), and therefore speaks against any substantial elevation of AN in twins. Furthermore, population-based studies have asserted that twins are considered representative of the general population in terms of the probability of acquiring a diagnosis of ASD (Curran et al., 2011; Hallmayer et al., 2002).

\section{Conclusion}

Our findings indicate that the screening assessment by parent-report of ASD may not be sufficient to detect elevation in autistic traits in girls who later develop AN, if those elevations are present. Instead, thorough clinical assessments are needed, and assessment needs to be tailored to capture variations in the ASD phenotype seen in females. In the acute phase of AN, individuals tend to display more autistic traits than before the onset of AN and after 
recovery from AN. This confirms previous concerns about diagnosing ASD during acute AN, as aspects of AN can mimic ASD. Future studies should use different measurements and methods in order to investigate whether autistic traits in AN are best conceptualized as an epiphenomenon of the acute AN phase (i.e., mimicking autistic symptoms) or whether these symptoms indeed represent ASD as a clinically verifiable neurodevelopmental disorder.

\section{Supporting information}

Additional supporting information may be found online in the Supporting Information section at the end of the article:

Table S1. Autism-tics, AD/HD, and other comorbidities inventory (A-TAC) - autism module at age 9 and age 18: item overview.

Table S2. Age at first diagnosis with anorexia nervosa (AN) and autism spectrum disorder (ASD) in the National Patient Register.

\section{Acknowledgements}

C.M.B. (521-2012-1754), P.L. (2016-01989), C.G. (5382013-8864), and S.L. (2017-02552) were supported by the Swedish Research Council (Vetenskapsrådet).
Furthermore, P.L. was supported by the Swedish Research Council for Health, Working Life and Welfare (2012-1678), C.G. by the Torsten Söderbergs Foundation (M151/14) and the AnnMari and Per Ahlqvist Foundation (2018), and S.L. by the SöderströmKönigska Foundation (SLS-566821). L.D. was supported by Queen Silvia's Jubilee Fund (2016) and The Sven Jerring Foundation (2018). The funders had no role in study design, data collection and analysis, decision to publish, or preparation of the manuscript. The authors gratefully acknowledge the contribution of the participants in the Child and Adolescent Twin Study in Sweden (CATSS) and their families. The authors acknowledge the Swedish Twin Registry for access to data. The Swedish Twin Registry is managed by Karolinska Institutet and receives funding through the Swedish Research Council under the grant no 201700641. C.M.B. reports Shire (grant recipient, Scientific Advisory Board member); Idorsia (consultant); and Pearson (author, royalty recipient). The remaining authors have declared that they have no competing or potential conflicts of interest.

\section{Correspondence}

Lisa Dinkler, Gillberg Neuropsychiatry Centre, Institute of Neuroscience and Physiology, University of Gothenburg, Kungsgatan 12, floor 2, 41119 Gothenburg, Sweden; Email: lisa.dinkler@gu.se

\section{Key points}

- The research of an association between anorexia nervosa (AN) and autism spectrum disorder (ASD) has been limited by cross-sectional and retrospective longitudinal studies.

- In a population-based twin sample, this study, for the first time, prospectively investigated childhood autistic traits in individuals who were later diagnosed with AN.

- Children with later AN did not show elevated autistic traits in childhood. Higher levels of autistic traits at age 18 in girls with acute or previous AN were confined to those with acute AN, confirming previous concerns about diagnosing ASD during acute AN.

- Future studies should examine whether autistic traits in AN are best conceptualized as an epiphenomenon of the acute AN phase or whether these symptoms indeed represent ASD as a clinically verifiable neurodevelopmental disorder.

\section{Notes}

1. The DSM-5 does not indicate a clear upper BMI limit for mild AN ('BMI $\geq 17 \mathrm{~kg} / \mathrm{m}^{2}$ '). Therefore, we chose to use the upper BMI limit for moderate AN ('BMI 16-16.99 kg/m²).

2. The required effect sizes were computed for onesided independent $t$-tests, while we used count models in the original analyses. T-tests yielded the same results for the main analyses as the count models did with regard to significance. Thus, the effect sizes presented here are likely very close to the results from power calculations for count models, which need to be done using simulation.
3. This value is obtained by multiplying Cohen's $d$ with the pooled standard deviation. The total ASD score ranges from 0 to $12 ; 1$ point corresponds to one item being endorsed with 'yes', and 0.5 points correspond to one item being endorsed with yes, to some extent'.

4. Does he/she... '(1) ...get absorbed by his/her interests in such a way as being repetitive or too intense? (2) ...get absorbed by routines in such a way as to produce problems for him/herself or others? (3) ...have specific body movements that appear automatically when he/she is happy or upset? (4) ...get absorbed by details'? 


\section{References}

Adamson, J., Leppanen, J., Murin, M., \& Tchanturia, K. (2018). Effectiveness of emotional skills training for patients with anorexia nervosa with autistic symptoms in group and individual format. European Eating Disorders Review, 26, 367-375.

American Psychiatric Association (2013). Diagnostic and statistical manual of mental disorders, 5th ed. Arlington, VA: American Psychiatric Association.

Anckarsäter, H., Hofvander, B., Billstedt, E., Gillberg, I.C., Gillberg, C., Wentz, E., \& Råstam, M. (2012). The sociocommunicative deficit subgroup in anorexia nervosa: Autism spectrum disorders and neurocognition in a community-based, longitudinal study. Psychological Medicine, 42, 1957-1967.

Anckarsäter, H., Lundström, S., Kollberg, L., Kerekes, N., Palm, C., Carlström, E., ... \& Lichtenstein, P. (2011). The Child and Adolescent Twin Study in Sweden (CATSS). Twin Research and Human Genetics, 14, 495-508.

Bentz, M., Jepsen, J.R., Pedersen, T., Bulik, C.M., Pedersen, L., Pagsberg, A.K., \& Plessen, K.J. (2017). Impairment of social function in young females with recent-onset Anorexia Nervosa and recovered individuals. Journal of Adolescent Health, 60, 23-32.

Carlin, J.B., Gurrin, L.C., Sterne, J.A., Morley, R., \& Dwyer, T. (2005). Regression models for twin studies: A critical review. International Journal of Epidemiology, 34, 1089-1099.

Claydon, E., \& Zullig, K.J. (2019). Eating disorders and academic performance among college students. Journal of American College Health, 68, 320-325.

Curran, S., Dworzynski, K., Happe, F., Ronald, A., Allison, C., Baron-Cohen, S., . . \& B Bolton, P.F. (2011). No major effect of twinning on autistic traits. Autism Research, 4, 377-382.

Dahlenburg, S.C., Gleaves, D.H., \& Hutchinson, A.D. (2019). Anorexia nervosa and perfectionism: A meta-analysis. International Journal of Eating Disorders, 52, 219-229.

Dean, M., Harwood, R., \& Kasari, C. (2017). The art of camouflage: Gender differences in the social behaviors of girls and boys with autism spectrum disorder. Autism, 21, 678-689.

Faul, F., Erdfelder, E., Lang, A.-G., \& Buchner, A. (2007). $\mathrm{G}^{*}$ Power 3: A flexible statistical power analysis program for the social, behavioral, and biomedical sciences. Behavior Research Methods, 39, 175-191.

Gillberg, C., \& Råstam, M. (1992). Do some cases of anorexia nervosa reflect underlying autistic-like conditions? Behavioural Neurology, 5, 27-32.

Goodman, A., Heshmati, A., Malki, N., \& Koupil, I. (2014). Associations between birth characteristics and eating disorders across the life course: findings from 2 million males and females born in Sweden, 1975-1998. American Journal of Epidemiology, 179, 852-863.

Gould, J., \& Ashton-Smith, J. (2011). Missed diagnosis or misdiagnosis? Girls and women on the autism spectrum. Good Autism Practice (GAP), 12, 34-41.

Hallmayer, J., Glasson, E.J., Bower, C., Petterson, B., Croen, L. Grether, J., \& Risch, N. (2002). On the twin risk in autism. The American Journal of Human Genetics, 71, 941-946.

Hansson, S.L., Svanström Röjvall, A., Råstam, M., Gillberg, C., Gillberg, C., \& Anckarsäter, H. (2005). Psychiatric telephone interview with parents for screening of childhood autism tics, attention-deficit hyperactivity disorder and other comorbidities (A-TAC): Preliminary reliability and validity. The British Journal of Psychiatry, 187, 262-267.

Hiller, R.M., Young, R.L., \& Weber, N. (2014). Sex differences in autism spectrum disorder based on DSM-5 criteria: Evidence from clinician and teacher reporting. Journal of Abnormal Child Psychology, 42, 1381-1393.

Hull, L., Lai, M.C., Baron-Cohen, S., Allison, C., Smith, P., Petrides, K.V., \& Mandy, W. (2019). Gender differences in self-reported camouflaging in autistic and non-autistic adults. Autism 24, 352-363.
Hull, L., Petrides, K.V., Allison, C., Smith, P., Baron-Cohen, S., Lai, M.C., \& Mandy, W. (2017). "Putting on my best normal": social camouflaging in adults with autism spectrum conditions. Journal of Autism and Developmental Disorders, 47, 2519-2534.

Idring, S., Rai, D., Dal, H., Dalman, C., Sturm, H., Zander, E., .., \& Magnusson, C. (2012). Autism spectrum disorders in the Stockholm Youth Cohort: Design, prevalence and validity. PLoS ONE, 7, e41280.

Keski-Rahkonen, A., \& Mustelin, L. (2016). Epidemiology of eating disorders in Europe: Prevalence, incidence, comorbidity, course, consequences, and risk factors. Current Opinion in Psychiatry, 29, 340-345.

Kinnaird, E., Norton, C., Stewart, C., \& Tchanturia, K. (2019). Same behaviours, different reasons: What do patients with co-occurring anorexia and autism want from treatment? International Review of Psychiatry, 31, 308-317.

Kirkovski, M., Enticott, P.G., \& Fitzgerald, P.B. (2013). A review of the role of female gender in autism spectrum disorders. Journal of Autism and Developmental Disorders, 43, 2584-2603.

Koch, S.V., Larsen, J.T., Mouridsen, S.E., Bentz, M., Petersen, L., Bulik, C., ... \& Plessen, K.J. (2015). Autism spectrum disorder in individuals with anorexia nervosa and in their first- and second-degree relatives: Danish nationwide register-based cohort-study. British Journal of Psychiatry, 206, $401-407$.

Kopp, S., \& Gillberg, C. (2011). The Autism Spectrum Screening Questionnaire (ASSQ)-Revised Extended Version (ASSQ-REV): An instrument for better capturing the autism phenotype in girls? A preliminary study involving 191 clinical cases and community controls. Research in Developmental Disabilities, 32, 2875-2888.

Lai, M.-C., Lombardo, M.V., Ruigrok, A.N.V., Chakrabarti, B., Auyeung, B., Szatmari, P., ... \& Baron-Cohen, S. (2016). Quantifying and exploring camouflaging in men and women with autism. Autism, 21, 690-702.

Larson, T., Anckarsäter, H., Gillberg, C., Ståhlberg, O., Carlström, E., Kadesjö, B., .. \& \& Gillberg, C. (2010). The Autism Tics, AD/HD and other Comorbidities inventory (A-TAC): Further validation of a telephone interview for epidemiological research. BMC Psychiatry, 10, 1.

Larson, T., Lundström, S., Nilsson, T., Selinus, E., Råstam, M., Lichtenstein, P., . . \& Kerekes, N. (2013). Predictive properties of the A-TAC inventory when screening for childhoodonset neurodevelopmental problems in a population-based sample. BMC Psychiatry, 13, 233.

Ludvigsson, J.F., Andersson, E., Ekbom, A., Feychting, M., Kim, J.L., Reuterwall, C., .. \& Olausson, P.O. (2011). External review and validation of the Swedish national inpatient register. BMC Public Health, 11, 450.

Lundström, S., Mårland, C., Kuja-Halkola, R., Anckarsäter, H., Lichtenstein, P., Gillberg, C., \& Nilsson, T. (2019). Assessing autism in females: The importance of a sex-specific comparison. Psychiatry Research, 282, 112566.

Mandy, W., Pellicano, L., St Pourcain, B., Skuse, D., \& Heron, J. (2018). The development of autistic social traits across childhood and adolescence in males and females. Journal of Child Psychology and Psychiatry and Allied Disciplines, 59, 1143-1151.

Mandy, W., \& Tchanturia, K. (2015). Do women with eating disorders who have social and flexibility difficulties really have autism? A case series. Molecular Autism, 6, 6.

Mårland, C., Lichtenstein, P., Degl'innocenti, A., Larson, T., Råstam, M., Anckarsäter, H., .. \& \& Lundström, S. (2017). The autism-tics, ADHD and other Comorbidities inventory (A-TAC): Previous and predictive validity. BMC Psychiatry, 17, 403.

Micali, N., Hagberg, K.W., Petersen, I., \& Treasure, J.L. (2013). The incidence of eating disorders in the UK in 2000-2009: findings from the General Practice Research Database. British Medical Journal Open, 3, e002646. 
Micali, N., Martini, M.G., Thomas, J.J., Eddy, K.T., Kothari, R., Russell, E., .. \& \& Treasure, J. (2017). Lifetime and 12-month prevalence of eating disorders amongst women in mid-life: A population-based study of diagnoses and risk factors. BMC Medicine, 15, 12.

Pooni, J., Ninteman, A., Bryant-Waugh, R., Nicholls, D., \& Mandy, W. (2012). Investigating autism spectrum disorder and autistic traits in early onset eating disorder. International Journal of Eating Disorders, 45, 583-591.

Postorino, V., Scahill, L., De Peppo, L., Fatta, L.M., Zanna, V., Castiglioni, M.C., .. \& \& Mazzone, L. (2017). Investigation of autism spectrum disorder and autistic traits in an adolescent sample with anorexia nervosa. Journal of Autism and Developmental Disorders, 47, 1051-1061.

$\mathrm{R}$ Core Team. (2019). R: A language and environment for statistical computing. Vienna, Austria: R Foundation for Statistical Computing. Available from: https://www.R-pro ject.org/ [last accessed 25 May 2020].

Rhind, C., Bonfioli, E., Hibbs, R., Goddard, E., Macdonald, P., Gowers, S., ... \& Treasure, J. (2014). An examination of autism spectrum traits in adolescents with anorexia nervosa and their parents. Molecular Autism, 5, 56.

Rück, C., Larsson, K.J., Lind, K., Perez-Vigil, A., Isomura, K., Sariaslan, A., ... \& Mataix-Cols, D. (2015). Validity and reliability of chronic tic disorder and obsessive-compulsive disorder diagnoses in the Swedish National Patient Register. British Medical Journal Open, 5, e007520.

Rynkiewicz, A., Schuller, B., Marchi, E., Piana, S., Camurri, A., Lassalle, A., \& Baron-Cohen, S. (2016). An investigation of the 'female camouflage effect' in autism using a computerized ADOS-2 and a test of sex/gender differences. Molecular Autism, 7, 10

Statacorp, (2017a). Stata longitudinal-data/panel-data reference manual: Release 15. College Station, TX: Stata Press.

Statacorp, (2017b). Stata statistical software: Release 15. College Station, TX: StataCorp LLC.

Stewart, C.S., Mcewen, F.S., Konstantellou, A., Eisler, I., \& Simic, M. (2017). Impact of ASD traits on treatment outcomes of eating disorders in girls. European Eating Disorders Review, 25, 123-128.

Sundquist, J., Ohlsson, H., Winkleby, M.A., Sundquist, K., \& Crump, C. (2016). School achievement and risk of eating disorders in a Swedish National Cohort. Journal of the American Academy of Child and Adolescent Psychiatry, 55, 41-46.e41.

Tchanturia, K., Adamson, J., Leppanen, J., \& Westwood, H. (2019). Characteristics of autism spectrum disorder in anorexia nervosa: A naturalistic study in an inpatient treatment programme. Autism, 23, 123-130.

Tchanturia, K., Doris, E., Mountford, V., \& Fleming, C. (2015). Cognitive Remediation and Emotion Skills Training (CREST) for anorexia nervosa in individual format: Self-reported outcomes. BMC Psychiatry, 15, 53.

Tchanturia, K., Larsson, E., \& Adamson, J. (2016). How anorexia nervosa patients with high and low autistic traits respond to group Cognitive Remediation Therapy. BMC Psychiatry, 16, 334.
Tchanturia, K., Lounes, N., \& Holttum, S. (2014). Cognitive remediation in anorexia nervosa and related conditions: A systematic review. European Eating Disorders Review, 22, 454-462.

Treasure, J. (2013). Coherence and other autistic spectrum traits and eating disorders: building from mechanism to treatment: The Birgit Olsson lecture. Nordic Journal of Psychiatry, 67, 38-42.

Treasure, J., \& Schmidt, U. (2013). The cognitive-interpersonal maintenance model of anorexia nervosa revisited: a summary of the evidence for cognitive, socio-emotional and interpersonal predisposing and perpetuating factors. Journal of Eating Disorders, 1, 13.

Trubanova, A., Donlon, K., Kreiser, N.L., Ollendick, T.H., \& White, S.W. (2013). Underidentification of autism spectrum disorder in females: A case series illustrating the unique presentation of this disorder in young women. Scandinavian Journal of Child and Adolescent Psychiatry and Psychology, 2, 66-76.

Van Wijngaarden-Cremers, P.J., Van Eeten, E., Groen, W.B., Van Deurzen, P.A., Oosterling, I.J., \& Van Der Gaag, R.J. (2014). Gender and age differences in the core triad of impairments in autism spectrum disorders: A systematic review and meta-analysis. Journal of Autism and Developmental Disorders, 44, 627-635.

Watson, H.J., Yilmaz, Z., Thornton, L.M., Hubel, C., Coleman, J.R.I., Gaspar, H.A., .. \& \& Bulik, C.M. (2019). Genome-wide association study identifies eight risk loci and implicates metabo-psychiatric origins for anorexia nervosa. Nature Genetics, 51, 1207-1214.

Westwood, H., Eisler, I., Mandy, W., Leppanen, J., Treasure, J., \& Tchanturia, K. (2016). Using the autism-spectrum quotient to measure autistic traits in anorexia nervosa: A systematic review and meta-analysis. Journal of Autism and Developmental Disorders, 46, 964-977.

Westwood, H., Mandy, W., Simic, M., \& Tchanturia, K. (2018). Assessing ASD in adolescent females with anorexia nervosa using clinical and developmental measures: A Preliminary Investigation. Journal of Abnormal Child Psychology, 46, 183-192.

Westwood, H., Mandy, W., \& Tchanturia, K. (2017). Clinical evaluation of autistic symptoms in women with anorexia nervosa. Molecular Autism, 8, 12.

Westwood, H., \& Tchanturia, K. (2017). Autism spectrum disorder in anorexia nervosa: An updated literature review. Current Psychiatry Reports, 19, 41.

Zerwas, S., Larsen, J.T., Petersen, L., Thornton, L.M., Mortensen, P.B., \& Bulik, C.M. (2015). The incidence of eating disorders in a Danish register study: Associations with suicide risk and mortality. Journal of Psychiatric Research, $65,16-22$.

Zucker, N.L., Losh, M., Bulik, C.M., Labar, K.S., Piven, J., \& Pelphrey, K.A. (2007). Anorexia nervosa and autism spectrum disorders: Guided investigation of social cognitive endophenotypes. Psychological Bulletin, 133, 976-1006.

Accepted for publication: 27 April 2020 